TRANSACTIONS OF THE

AMERICAN MATHEMATICAL SOCIETY

Volume 196, 1974

\title{
THE HOMOTOPY TYPE OF THE SPACE OF DIFFEOMORPHISMS. II
}

BY

\section{DAN BURGHELEA(1) AND RICHARD LASHOF(2)}

ABSTRACT. The result (proved in Part I) that $\operatorname{Diff}\left(D^{n}, \partial\right) \simeq$ $\Omega^{n+1}\left(\mathrm{PL}_{n} / O_{n}\right)$ is used to compute some new homotopy of $\operatorname{Diff}\left(D^{n}, \partial D^{n}\right)$. The relation between smooth and PL pseudo-isotopy is explored. Known and new results on the homotopy of $\mathrm{PL}_{n}$ are summarized.

5. Some remarks on $\mathrm{PL}_{n}$ and $\mathrm{Top}_{n}$. We first summarize known results. In low dimensions we have the following are contractible:

(1) $\mathrm{PL}_{1} / O_{1} \simeq \mathrm{PL}_{2} / O_{2} \simeq *$, $\operatorname{Top}_{1} / O_{1} \simeq \operatorname{Top}_{2} / O_{2} \simeq *$.

It is trivial to show that $\mathrm{PL}_{1} / O_{1} \simeq \operatorname{Top}_{1} / O_{1} \simeq *$. Kneser [16] showed a long time ago that $\operatorname{Top}_{2} / \mathrm{O}_{2} \simeq *$. That $\mathrm{PL}_{2} / \mathrm{O}_{2} \simeq *$ has been shown recently by $\Lambda$ kiba and P. Scott [1].

In the "stable" range, we recall the results of Haefliger and Wall [26a]:

(2) $\pi_{i}\left(\mathrm{PL}_{k+1}, \mathrm{PL}_{k}\right)=0$ for $i \leq k-1$, and

$\left(2^{\prime}\right) \operatorname{Ker}\left(\pi_{k-1}\left(\mathrm{PL}_{k}\right) \rightarrow \pi_{k-1}\left(\mathrm{PL}_{k+1}\right)\right)=\operatorname{Image} i_{*}^{k}\left(\operatorname{Ker}\left(\pi_{k-1}\left(O_{k}\right) \rightarrow\right.\right.$ $\left.\left.\pi_{k-1}\left(O_{k+1}\right)\right)\right), i^{k}: O_{k} \rightarrow \mathrm{PL}_{k}$ the natural map.

By standard smoothing the ory [17],

(3) $\pi_{i}\left(\mathrm{PL}_{k} / O_{k}\right) \rightarrow \pi_{i}(\mathrm{PL} / O)$ is bijective for $i \leq k$.

By Theorem 4.6 (see also Hirsch [38]),

(4) $\pi_{n+1}\left(\mathrm{PL}{ }_{n} / O_{n}\right) \rightarrow \pi_{n+1}(\mathrm{PL} / 0)$ is bijective, $n \geq 5$.

$\left(4^{\prime}\right) \pi_{n+2}\left(\mathrm{PL}_{n} / 0_{n}\right) \rightarrow \pi_{n+2}(\mathrm{PL} / 0)$ is surjective, $n \geq 5$.

Combining these results we get

Theorem 5.1. (a) $\pi_{i}\left(\mathrm{PL}_{k+1} / O_{k+1}, \mathrm{PL}_{k} / O_{k}\right)=0$ for $i \leq k+2, k \geq 5$.

(b) $\pi_{i}\left(\operatorname{Top}_{k+1} / O_{k+1}, \operatorname{Top}_{k} / O_{k}\right)=0$ for $i \leq k+2, k \geq 5$.

Part (b) follows from (a) and the results of Kirby and Siebenmann [15].

Received by the editors June 13, 1973 and, in revised form, September 14, 1973. AMS (MOS) subject classifications (1970). Primary 57D50, 57D40, 57A35, 57E05.

(1) During the preparation of this work the first named author was partially supported by Aarhus Universitet-Math. Inst. and by Sonderforschungsbereich Theoretische Mathematik an der Universität Bonn.

(2) The second named author was partially supported by the National Science Foundation, USA. 
Recently, Volodin [35] has defined Whitehead groups $\mathrm{Wh}^{0}(G), \mathrm{Wh}^{1}(G)$, $W_{h}{ }^{2}(G), \cdots$ for any group $G$, with $W_{h}{ }^{0}(G)=\widetilde{K}_{0}(G), W_{h}{ }^{1}(G)=W h(G)$ and $W_{h}{ }^{2}(G)$ being the same as the algebraic Whitehead group introduced by Hatcher and Wagoner [12]. Volodin obtains the results of Hatcher and Wagoner on $\pi_{0}\left(C^{d}(M, \partial)\right)$ and also claims $\pi_{1}(C(M, \partial)) \simeq Z_{2} \oplus \mathbb{W h}^{3}(0)$, where $C^{d}(M, \partial)=$ group of concordances (i.e., pseudo-isotopies) of $M \bmod \partial$; for $M$ 2-connected and $\operatorname{dim} M$ sufficiently large. This last result has also been obtained by Hatcher. In particular, Volodin claims

$$
\pi_{1}\left(C^{d}\left(D^{k-1}, \partial\right)\right)=\pi_{1}\left(\operatorname{Diff}\left(D^{k}, D_{+}^{k-1}\right)\right) \simeq Z_{2} \oplus \mathrm{Wh}^{3}(0)
$$

for $k \geq 8$. From Theorem 4.4(c) we therefore have

Theorem 5.1. ( $\left.\mathrm{a}^{\prime}\right) \pi_{k+3}\left(\mathrm{PL}_{k+1} / O_{k+1}, \mathrm{PL}_{k} / O_{k}\right) \simeq Z_{2} \oplus \mathrm{Wh}^{3}(0), k \geq 7$.

(b') $\pi_{k+3}\left(\operatorname{Top}_{k+1} / O_{k+1}, \operatorname{Top}_{k} / O_{k}\right) \simeq Z_{2} \oplus \mathbb{W h}^{3}(0), k \geq 7$.

Corollary 5.2. (a) $\pi_{i}\left(\mathrm{PL} / O, \mathrm{PL}_{n} / O_{n}\right)=0$ for $i \leq n+2, n \geq 5$ and $\pi_{n+3}\left(\mathrm{PL} / O, \mathrm{PL}_{n} / O_{n}\right)=Z_{2} \oplus \mathrm{Wh}^{3}(0)$ if $n \geq 7$.

(b) $\pi_{i}\left(\mathrm{Top} / O, \operatorname{Top}_{n} / O_{n}\right)=0$ for $i \leq n+2, n \geq 5$ and $\pi_{n+3}\left(\operatorname{Top} / O, \operatorname{Top}_{n} / O_{n}\right)=$ $z_{2} \oplus \mathbb{W h}^{3}(0)$ if $n \geq 7$.

Since $\pi_{i}\left(\mathrm{PL}_{k+1} / O_{k+1}, \mathrm{PL}_{k} / O_{k}\right) \simeq \pi_{i}\left(\mathrm{PL}_{k+1} / \mathrm{PL}_{k}, O_{k+1} / O_{k}\right)$, we have

Theorem 5.1'. $\pi_{i}\left(\mathrm{PL}_{k+1} / \mathrm{PL}_{k}, O_{k+1} / O_{k}\right) \simeq 0$ for $i \leq k+2, k \geq 5$, and $\pi_{k+3}\left(\mathrm{PL}_{k+1} / \mathrm{PL}_{k}, O_{k+1} / O_{k}\right) \simeq Z_{2} \oplus \mathrm{Wh}^{3}(O), k \geq 7$. (Similarly for Top.)

The main result of [39] gives a fibration $\mathrm{PL}_{k} \subset \mathrm{PL}_{k+1} \rightarrow S^{k} \times B C^{p l}\left(S^{k}\right)$, where $C^{p l}\left(S^{k}\right)$ is the group of PL pseudo-isotopies of $S^{k+1}$ and $B C^{p l}\left(S^{k}\right)$ is the universal base space. Since the fibration $O_{k} \rightarrow O_{k+1} \rightarrow S^{k}$ maps into the above fibration, we have that

Proposition 5.3. $\pi_{i}\left(O_{k+1} / O_{k}\right) \rightarrow \pi_{i}\left(\mathrm{PL}_{k+1} / \mathrm{PL}_{k}\right)$ is split injective, all $i, k$ (i.e., $\mathrm{PL}_{k+1} / \mathrm{PL} \mathrm{L}_{k} \simeq O_{k+1} / O_{k} \times B C^{p l}\left(S^{k}\right)$.

Before proceeding further, we quote other known results:

(5) $\pi_{k}\left(O_{n}\right) \rightarrow \pi_{k}(O)$ is a split surjection for $k<2(n-1), n \geq 13$ [30].

(6) $\pi_{i}\left(O_{n}, O_{n-k}\right) \rightarrow \pi_{i}\left(G_{n}, G_{n-k}\right)$ is an isomorphism for all $i<2(n-k)-2$, where $G_{n}$ is the $H$-space of homotopy equivalences of $S^{n-1}$ with the $C \cdot O$ topology [36].

$(7) \pi_{i}(O) \rightarrow \pi_{i}(\mathrm{PL})$ and $\pi_{i}(O) \rightarrow \pi_{i}(\mathrm{Top})$ are injective. Also $\pi_{i}(\mathrm{PL} / O)$ and $\pi_{i}(\mathrm{Top} / 0)$ are finite [40].

(8) $\pi_{i}\left(O, O_{n}\right) \otimes Q \rightarrow \pi_{i}\left(G, G_{n}\right) \otimes Q$ is an isomorphism for $i \leq 2 n-2$ [36]. 
Proposition 5.4. (i) $\pi_{i}\left(0, O_{n}\right) \rightarrow \pi_{i}\left(\mathrm{PL}, \mathrm{PL}_{n}\right)$ and $\pi_{i}\left(O, O_{n}\right) \rightarrow \pi_{i}\left(\mathrm{Top}, \mathrm{Top}_{n}\right)$ are injective for all $i \leq 2 n-2$.

(ii) $\pi_{i}\left(O, O_{n}\right) \rightarrow \pi_{i}\left(\mathrm{PL}, \mathrm{PL}_{n}\right) \simeq \pi_{i}($ Top, Top $n)$ is bijective for $i \leq n+2$ and $n \geq 5$.

(iii) $\pi_{i}\left(O_{n}\right) \otimes Q \rightarrow \pi_{i}\left(\mathrm{PL}_{n}\right) \otimes Q$ and $\pi_{i}\left(O_{n}\right) \otimes Q \rightarrow \pi_{i}\left(\mathrm{Top}_{n}\right) \otimes Q$ are injective for all $i$.

(iv) If $n \geq 5, \pi_{i}\left(O_{n}\right) \rightarrow \pi_{i}\left(\mathrm{PL}_{n}\right)$ and $\pi_{i}\left(O_{n}\right) \rightarrow \pi_{i}\left(\mathrm{Top}_{n}\right)$ are injective for $i \leq n$ and also for $i=n+3$ if $n \equiv 1,5,7(\bmod 8)$ and $i=n+4$ if $n \equiv 0,4,5(\bmod 8)$.

Proof. (i) follows from (6), and (ii) follows from Corollary 5.2. For (iii) consider the commutative diagram

(9)

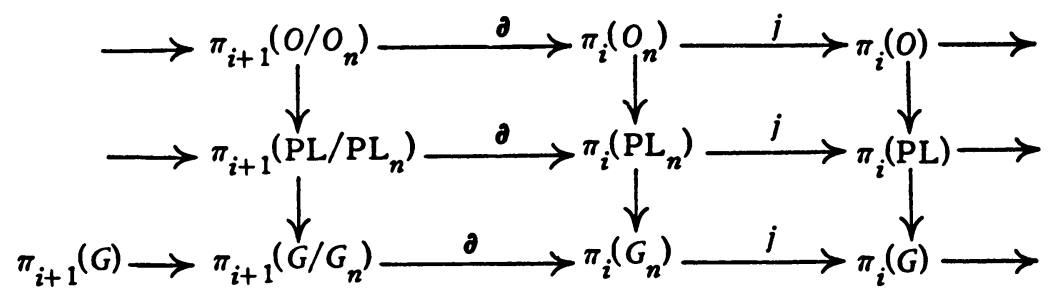

Since $\pi_{i}(G) \otimes Q=0$ all $i$, and $\pi_{i}(0) \otimes Q \rightarrow \pi_{i}(\mathrm{PL}) \otimes Q$ is an isomorphism by (7), it follows from (8) that $\pi_{i}\left(O_{n}\right) \otimes Q \rightarrow \pi_{i}\left(\mathrm{PL}_{n}\right) \otimes Q$ is injective for $i \leq 2 n-3$. But $\pi_{i}\left(O_{n}\right) \otimes Q=0$ for $i>2 n-3$.

For the first part of (iv) note first that for $i \leq n-2, \pi_{i}\left(O_{n}\right) \rightarrow \pi_{i}(0)$ is an isomorphism and hence by (7), $\pi_{i}\left(O_{n}\right) \rightarrow \pi_{i}\left(\mathrm{PL}_{n}\right)$ is a monomorphism. Now consider the commutative diagram:

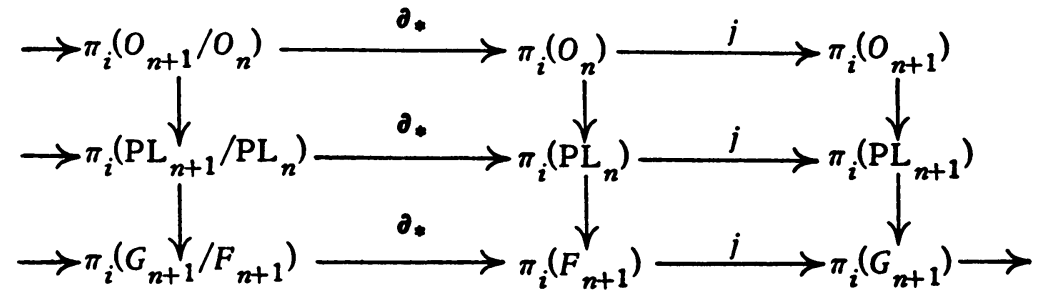

If $i=n-1$, and $\alpha \in \pi_{n-1}\left(O_{n}\right)$ is in $\operatorname{Ker} j=\operatorname{Im} \partial$, then $\alpha$ is either infinite cyclic or of order 2. The first case is covered by (iii), and in the second case it maps nontrivially into $\pi_{n-1}\left(F_{n+1}\right)$, since for $i_{n}$ the generator of $\pi_{n}\left(S^{n}\right)=$ $\pi_{n}\left(G_{n+1} / F_{n+1}\right), \partial i_{n} \in \pi_{n-1}\left(F_{n+1}\right)$ is nontrivial (except for $\left.n=1,3,7\right)$. Hence in either case $\operatorname{Ker} j \subset \pi_{n-1}\left(O_{n}\right)$ maps monomorphically into $\pi_{n-1}\left(\mathrm{PL}_{n}\right)$; and if $\alpha \in \pi_{n-1}\left(O_{n}\right)$ maps nontrivially under $j$, it maps nontrivially into $\pi_{n-1}\left(\mathrm{PL}{ }_{n}\right)$ by the first part of the argument.

For $i=n$ and $n \equiv 3(\bmod 4)$ the result follows from the fact that $\pi_{n+1}\left(O / O_{n}\right)=0[33]$. 
If $n \neq 3(\bmod 4)$, let $\eta_{n} \in \pi_{n+1}\left(S^{n}\right)=Z_{2}$, the generator. Then under the map $\pi_{n+1}\left(S^{n}\right) \stackrel{\partial}{\rightarrow} \pi_{n}\left(F_{n+1}\right) \simeq \pi_{2 n}\left(S^{n}\right), \eta_{n}$ goes into the Whitehead product $\left[\eta_{n}, i_{n}\right] \neq 0$ [32]. Combining this $w$ ith the injectivity of $\pi_{n}\left(O_{n+1}\right) \rightarrow \pi_{n}\left(\mathrm{PL}_{n+1}\right)$, the result follows.

Finally, the second part of (iv) follows because for $n \geq 4, \pi_{i+n}\left(0, O_{n}\right)=0$ for $i=4$ and $n \equiv 1,5,7(\bmod 8)$, or for $i=5$ and $n \equiv 0,4,5(\bmod 8)$, see [33].

The argument for $\operatorname{Top}_{n}$ is identical.

Corollary 5.5. If $n \geq 5$ and $i \leq n+1, \pi_{i}\left(\mathrm{PL}_{n}\right) \rightarrow \pi_{i}(\mathrm{PL})$ and $\pi_{i}\left(\operatorname{Top}_{n}\right) \rightarrow$ $\pi_{i}$ (Top) are surjective.

Proof. It suffices to show that $\pi_{i+1}\left(\mathrm{PL} / \mathrm{PL}_{n}\right) \rightarrow \pi_{i}\left(\mathrm{PL}_{n}\right)$ is injective for $i \leq n$. But this follows from Proposition 5.4(ii) and (iv), using diagram (9).

Now we compare $\mathrm{PL}_{n}$, Top $_{n}$ with the corresponding groups $\widetilde{\mathrm{PL}}_{n}, \widetilde{\mathrm{TOP}}_{n}$ for

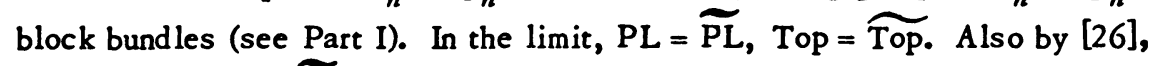
$\pi_{i}\left(O / O_{n}\right) \simeq \pi_{i}\left(\mathrm{PL} / \widetilde{\mathrm{PL}}{ }_{n}\right) \simeq \pi_{i}\left(\mathrm{Top} / \widetilde{\mathrm{Top}}_{n}\right) \simeq \pi_{i}\left(G / G_{n}\right)$ for $n \geq 5, i<2 n-2$.

Proposition 5.6. If $n \geq 5, \pi_{i}\left(\mathrm{PL}_{n}\right) \rightarrow \pi_{i}\left(\widetilde{\mathrm{PL}}{ }_{n}\right)$ and $\pi_{i}\left(\mathrm{Top}_{n}\right) \rightarrow \pi_{i}\left(\widetilde{\mathrm{Top}_{n}}\right)$ are isomorphisms for $i \leq n+1$ and epimorphisms for $i=n+2$.

Proof. Consider the commutative diagram

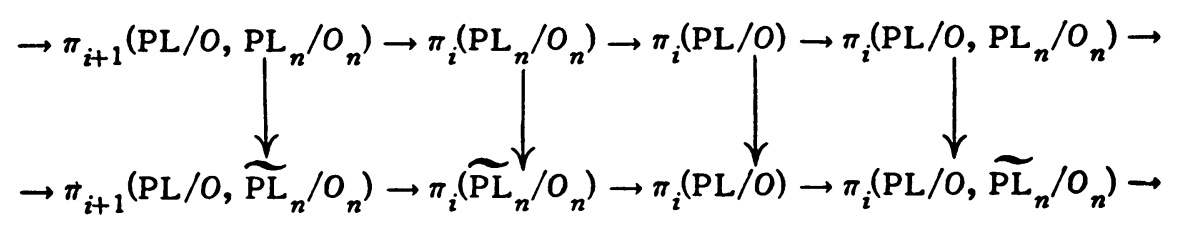

We have

$$
\begin{array}{ll}
\pi_{i}\left(\mathrm{PL} / O, \widetilde{\mathrm{PL}}_{n} / O_{n}\right) \cong \pi_{i}\left(\mathrm{PL} / \widetilde{\mathrm{PL}}_{n}, 0 / O_{n}\right)=0 & \text { for } i<2 n-2 \\
\pi_{i}\left(\mathrm{PL} / O, \mathrm{PL}_{n} / O_{n_{0}}\right) \cong \pi_{i}\left(\mathrm{PL} / \mathrm{PL}{ }_{n}, 0 / O_{n}\right)=0 & \text { for } i \leq n+2
\end{array}
$$

by 5.2. Hence $\pi_{i}\left(\mathrm{PL}_{n} / O_{n}\right) \rightarrow \pi_{i}\left(\widetilde{\mathrm{PL}}_{n} / O_{n}\right)$ is an isomorphism for $i \leq n+1$ and an epimorphism for $i=n+2$. The result follows.

Corollary 5.7. If $n \geq 5$ and $M^{n} \subset M^{n+k}$ is a PL embedding of PL manifolds, then $M^{n}$ bas a PL normal bundle if $n \leq k+3$, which is unique if $n \leq k+2$.

Conjecture. ( $\left.{ }^{3}\right) \pi_{i}\left(O_{n}\right) \rightarrow \pi_{i}\left(\mathrm{PL}_{n}\right)$ is injective for all $i<2 n-3$.

We note that the kernel is at most 2-primary. This is well known and we give a simple argument: In fact, $\pi_{i}\left(G_{n}\right) \rightarrow \pi_{i}(G)$ is an epimorphism for $i \leq 2 n-3$, $\bmod$

(3) A. Campo informs us that he can prove $\pi_{i}\left(O_{n}\right) \rightarrow \pi_{i}\left(\mathrm{PL}{ }_{n}\right)$ is injective for $i \leq n+2$. 
the 2-primary component. Hence from the diagram

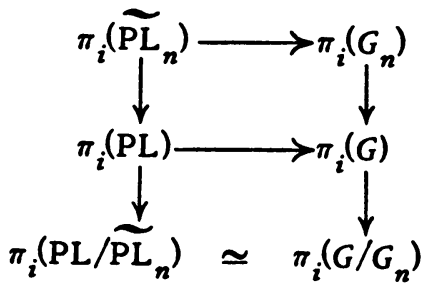

we see that $\pi_{i}\left(\widetilde{P L}_{n}\right) \rightarrow \pi_{i}(\mathrm{PL})$ is an epimorphism mod the 2-primary component. But then from the diagram

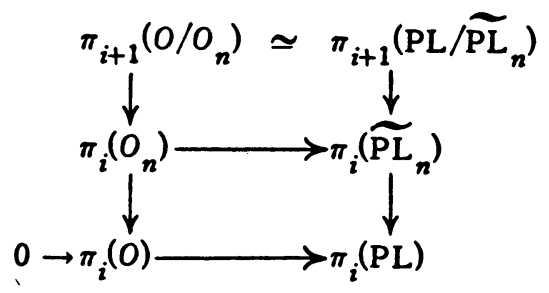

we see that $\pi_{i}\left(O_{n}\right) \rightarrow \pi_{i}\left(\widetilde{P L}_{n}\right)$ is injective $\bmod 2$-primary for $i<2 n=3$, and $a$ fortiori, the same holds for $\pi_{i}\left(O_{n}\right) \rightarrow \pi_{i}\left(\mathrm{PL}_{n}\right)$.

6. Isotopy and pseudo-isotopy. If $M^{n}$ is a compact differentiable manifold and $t:\left|M^{n}\right| \rightarrow M^{n}$ a differentiable triangulation; let $C^{d}(M, \partial), C^{p l}(M, \partial), C^{t}(M, \partial)$ denote the groups

$$
\operatorname{Diff}(M \times I ; \partial M \times l \cup M \times\{0\}), \quad \operatorname{PL}(|M| \times J, \partial|M| \times I \cup|M| \times\{0\}),
$$

$$
\operatorname{Homeo}(M \times I ; \partial M \times I \cup M \times\{0\})
$$

respectively. (In the differentiable case one needs to "smooth the corners" of $M \times I$ if $\partial M \neq \varnothing$.) These groups are the so-called concordance or pseudo-isotopy groups.

Theorem 6.1. If $M^{n}$ is a compact connected differentiable manifold, then $\pi_{i}\left(C^{d}(M, \partial)\right) \rightarrow \pi_{i}\left(C^{p l}(M, \partial)\right)$ is an isomorphism for $i=0$ and an epimorpbism for $i=1$.

In particular, this the orem says that a differentiable pseudo-isotopy of $(M, \partial M)$ may be deformed into an isotopy if and only if it may be so deformed as a piecewise differentiable pseudo-isotopy.

Theorem 6.2. If $M^{n}$ is a compact connected piecewise linear manifold witb $n \geq 5$, then $\pi_{i}\left(C^{p l}(M, \partial)\right) \rightarrow \pi_{i}\left(C^{t}(M, \partial)\right)$ is an isomorphism, all $i$. 
To prove these theorems, we will apply the main theorem (Theorem 4.2)(4) of Part $\mathrm{I}$, to show that $\pi_{i}\left(C^{p l}(M, \partial)\right) / C^{d}(M, \partial)=0$ for $i=0,1$ and $\pi_{i}\left(C^{t}(M, \partial)\right) /\left(C^{p l}(M, \partial)\right)=0$, all $i$. For this purpose we need some elementary lemmas on the space of sections of a fibre bundle which we state without proof.

Lemma 6.3. Let $p: P \rightarrow B$ be a fibre bundle with connected fibre $F$. Let $\Gamma(P)=$ space of sections of $P$. Suppose given a fixed section $s_{0}: B \rightarrow P$. Then

(a) If $p$ is trivial, $\Gamma(P)=F^{B}$ and we bave a split fibration

$$
(F, *)^{(B, *)} \rightarrow F^{B} \leadsto F \text {. }
$$

(b) If $B$ is a suspension of a CW-complex, we bave a fibration

$$
(F, *)^{(B, *)} \rightarrow \Gamma(P) \rightarrow F .
$$

(c) If $F$ is k-connected and $B$ is a CW-complex with $\operatorname{dim} B=j$, then $\pi_{i}(\Gamma(P))=$ 0 for $i+j \leq k$.

Lemma 6.4. Let $p: P \rightarrow B$ be a fibre bundle with connected fibre $F$, and $p_{1}: P_{1} \rightarrow B$ a subbundle $P_{1} \subset P$ with connected fibre $F_{1} \subset F$. Let $\Gamma^{\partial 0}\left(P \times I, P_{1}\right)=$ space of sections $s$ of $P \times 1: P \times I \rightarrow B \times I$ sucb that $s \mid B \times 0=$ $s_{0}$ and $s(B \times 1) \subset P_{1} \times 1$. Then fibration:

(a) If $\left(p, p_{1}\right)$ is trivial, $\Gamma^{\partial 0}\left(P \times I, P_{1}\right)=E\left(F, F_{1}\right)^{B}$ and we bave the split

$$
\left(E\left(F, F_{1}\right), *\right)^{(B, *)} \rightarrow\left(E\left(F, F_{1}\right)\right)^{B} \rightarrow E\left(F, F_{1}\right) .
$$

(Here $E\left(F, F_{1}\right)$ is the space of paths in $E$ from base point to $F_{1}$. )

(b) If $B$ is a suspension of a CW-complex, we get a fibration

$$
\left(E\left(F, F_{1}\right), *\right)^{(B, *)} \rightarrow \Gamma^{\partial_{0}}\left(P \times I, P_{1}\right) \rightarrow E\left(F, F_{1}\right) .
$$

In particular, if $P_{1}=s_{0}(B)$, then we get a fibration

$$
(\Omega(F), *)^{(B, *)} \rightarrow \Gamma^{\partial}\left(P \times I, P_{1}\right) \rightarrow \Omega F
$$

(c) If $\pi_{i}\left(E\left(F, F_{1}\right)\right) \simeq \pi_{i+1}\left(F, F_{1}\right)=0$ for $i \leq k$, and $\operatorname{dim} B=j$, then $\pi_{i}\left(\Gamma^{\partial} 0\left(P, P_{1}\right)\right)=0$ for $i+j \leq k$.

Proof of 6.1. By Theorem 4.2 of Part I,

$$
C^{p l}(M, \partial) / C^{d}(M, \partial)=\Gamma^{\partial_{0}}\left(P^{p l} \times l, \partial P^{p l}, s_{0}\right),
$$

where the fibre of $P^{p l}$ is $\mathrm{PL}_{n+1} / O_{n+1}$ and the fibre of $\partial P^{p l}$ is $\mathrm{PL}_{n} / O_{n}$. By Theorem 5.1' we have

(4) We use the Top-PL version of Theorem 4.2 to prove Theorem 6.2. 


$$
\pi_{i}\left(E\left(\mathrm{PL}_{n+1} / O_{n+1}, \mathrm{PL}_{n} / O_{n}\right)\right)=0 \text { for } i \leq n+1 .
$$

Hence by Lemma $6.4(\mathrm{c}), \pi_{i}\left(C^{p l}(M, \partial) / C^{d}(M, \partial)\right)=0$ for $i=0,1$.

Proof of 6.2. The argument is the same, except in this case

$$
\pi_{i}\left(\operatorname{Top}_{n+1} / \mathrm{PL}_{n+1}, \operatorname{Top}_{n} / \mathrm{PL}_{n}\right)=0 \text { all } i \text { if } n \geq 5 \text {. }
$$

We now consider the special case of $C^{d}\left(S^{n}\right)$ and $C^{p l}\left(S^{n}\right)$.

By Proposition 5.3,

$$
C^{p l}\left(S^{n}\right) \simeq E\left(\mathrm{PL}_{n+1} / O_{n+1}, \mathrm{PL}_{n} / O_{n}\right) .
$$

Hence by Theorem $5.1^{\prime}$,

$$
\begin{aligned}
\pi_{i}\left(C^{p l}\left(S^{n}\right)\right) & =0 \text { for } i \leq n+1, n \geq 5 \\
& =Z_{2}+W^{3}(0) \text { for } i=n+2, n \geq 7 .
\end{aligned}
$$

On the other hand, Chenciner [37] has shown that $\operatorname{Diff}\left(D^{n+1}, D_{+}^{n}\right) \simeq C^{d}\left(S^{n}\right)$ and hence by Theorem 4.4

$$
C^{d}\left(S^{n}\right) \simeq \Omega^{n+1}\left(E\left(\mathrm{PL}_{n+1} / O_{n+1}, \mathrm{PL}_{n} / O_{n}\right)\right)
$$

and hence by (1) and (3) with $C_{n}^{d}=C^{d}\left(S^{n}\right), C_{n}^{p l}=C^{p l}\left(S^{n}\right)$.

Proposition 6.5 (Chenciner). $\pi_{i}\left(C_{n}^{d}\right) \simeq \pi_{i+n+1}\left(C_{n}^{p l}\right)$, all $i$.

Thus we see that, although $\pi_{0}\left(C^{p l}(M, \partial)\right) \simeq \pi_{0}\left(C^{d}(M, \partial)\right), \pi_{1}\left(C^{p l}(M, \partial)\right) \neq$ $\pi_{1}\left(C^{d}(M, \partial)\right)$ in general. The relationship between PL and Diff pseudo-isotopy groups will be explored in a forthcoming paper with $M$. Rothenberg.

Finally, we consider the implications of these results for diffeomorphisms of the disc: First Volodin and Hatcher have pointed out that the split fibration $C^{d}\left(S^{n-1}\right) \rightarrow$ Diff $D^{n} \rightleftarrows S O_{n}$, together with their result on $\pi_{1}\left(C^{d}\left(S^{n-1}\right)\right)$ implies

$$
\pi_{0}\left(\text { Diff } D^{n}\right)=0 \text { and } \pi_{1}\left(\text { Diff } D^{n}\right) \simeq Z_{2}+Z_{2}+W_{h^{3}}(0)
$$

(We are considering only orientation preserving diffeomorphisms.)

To obtain information on $\operatorname{Diff}\left(D^{n}, \partial\right)$, we consider the fibration

$$
\operatorname{Diff}\left(D^{n+1}, \partial\right) \rightarrow \operatorname{Diff}\left(D^{n+1}, D_{+}^{n}\right) \rightarrow \operatorname{Diff}\left(D^{n}, \partial\right)
$$

and its homotopy sequence

$$
\begin{aligned}
\rightarrow \pi_{2}\left(\operatorname{Diff}\left(D^{n}, \partial\right)\right) & \rightarrow \pi_{1}\left(\operatorname{Diff}\left(D^{n+1}, \partial\right)\right) \rightarrow \pi_{1}\left(\operatorname{Diff}\left(D^{n+1}, D_{+}^{n}\right)\right) \rightarrow \pi_{1}\left(\operatorname{Diff}\left(D^{n}, \partial\right)\right) \\
& \rightarrow \pi_{0}\left(\operatorname{Diff}\left(D^{n+1}, \partial\right)\right) \rightarrow \pi_{0}\left(\operatorname{Diff}\left(D^{n+1}, D_{+}^{n}\right)\right) .
\end{aligned}
$$

Since $\pi_{0}\left(\operatorname{Diff}\left(D^{n+1}, D_{+}^{n}\right)\right)=0$ and $\pi_{0}\left(\operatorname{Diff}\left(D^{n+1}, \partial\right)\right)$ is Milnor's $\Gamma_{n+1}$ group and $\pi_{1}\left(\operatorname{Diff}\left(D^{n+1}, D_{+}^{n}\right)\right) \cong Z_{2}+W^{3}(0)$, we see that $\pi_{1}\left(\operatorname{Diff}\left(D^{n}, \partial\right)\right)$ is arbitrarily 
large, and hence is general $\pi_{1}\left(\operatorname{Diff}\left(D^{n+1}, \partial\right)\right) \rightarrow \pi_{1}\left(\operatorname{Diff}\left(D^{n+1}, D_{+}^{n}\right)\right)$ has a kernel. Hence $\pi_{2}\left(\operatorname{Diff}\left(D^{n}, \partial\right)\right) \neq 0$ in general.

7. A bilinear pairing and some new homotopy of $\operatorname{Diff}\left(D^{n}, \partial D^{n}\right)$. In this chapter, using the results of $\$ 4$, we will get some estimate on the homotopy groups of $\operatorname{Diff}\left(D^{n}, \partial D^{n}\right)$, Theorem 7.4. The methods of [2], [3] permit the use of these estimates to prove the nontriviality of $\pi_{i}\left(\operatorname{Diff}\left(M^{n}\right)\right.$ ) (see for instance Corollary 7.5). From now on we assume that we are in the category of basepointed spaces which have abelian fundamental groups.

Let us consider the map $\psi_{k, p}^{X}: \pi_{k}(X, *) \times \pi_{p}\left(S^{k}, s_{0}\right) \rightarrow \pi_{p}(X, *)$ defined by $\psi(\alpha, \beta)=\alpha_{\#}(\beta)$; more precisely, if $\alpha$ is a homotopy class $\left(s^{k}, s_{0}\right) \rightarrow(X, *)$ it induces a group homomorphism $\alpha$ for homotopy groups in dimension $p$, and we take as $\psi(a, \beta)$ the image of $\beta$ by this homomorphism. With this definition, it is clear that

$$
\psi_{k, p}^{X}\left(a, \beta_{1}+\beta_{2}\right)=\psi_{k, p}^{X}\left(a, \beta_{1}\right)+\psi_{k, p}^{X}\left(a, \beta_{2}\right)
$$

If we set $\dot{\pi}_{p}\left(S^{k}\right)=\left\{\beta \in \pi_{p}\left(S^{k}\right) \mid \beta \in \operatorname{Im} \Sigma\right\}$, where by $\Sigma$ we mean the suspension homomorphism, and if we consider the restriction of $\psi_{k, p}^{X}$ to $\pi_{k}(X, *) \times \cdot$ $\pi_{p}\left(S^{k}\right) \rightarrow \pi_{p}(X, *)$ we also have

$$
\psi_{k, p}^{X}\left(a_{1}+a_{2}, \beta\right)=\psi_{k, p}^{X}\left(a_{1}, \beta\right)+\psi_{k, p}^{X}\left(a_{2}, \beta\right)
$$

for any $\beta$. Indeed if $\beta \in \dot{\pi}_{p}\left(S^{k}\right)$, it can be represented by $\Sigma \beta^{\prime}: S^{p} \rightarrow S^{k}$ with $\beta^{\prime}:\left(S^{p-1}, s_{0}\right) \rightarrow\left(S^{k-1}, s_{0}\right)$; such a map induces always a group homomorphism $\left(\Sigma \beta^{\prime}\right)_{*}: \pi_{k}(X, *) \rightarrow \pi_{p}(X, *)$, and because $\psi_{k, p}^{X}(\alpha, \beta)=\left(\Sigma \beta^{\prime}\right)_{*}(\alpha)$ we have

$$
\psi_{k, p}^{X}\left(a_{1}+a_{2}, \beta\right)=\psi_{k, p}^{X}\left(a_{1}, \beta\right)+\psi_{k, p}^{X}\left(a_{2}, \beta\right) .
$$

Therefore we can extend $\psi_{k, p}^{X}$ to a unique group homomorphism $\bar{\psi}_{k, p}^{X}: \pi_{k}(X, *) \otimes$ $\pi_{p}\left(S^{k}, s_{0}\right) \rightarrow \pi_{k+p}(X, *)$. It is easy to check that $\bar{\psi}^{X} \ldots$ is natural with respect to $X$, i.e., if $f:(X, *) \rightarrow(Y, *)$ is a continuous map, we have the following commutative diagram

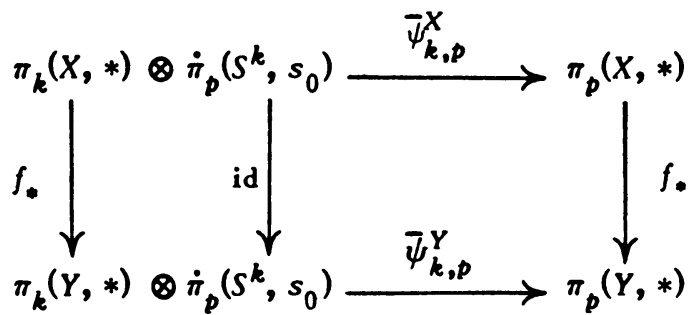

We also notice that for $p \leq 2 k-2, \pi_{p}\left(S^{k}, s_{0}\right)=\pi_{p+1}\left(S^{k+1}, s_{0}\right)=\pi_{p-k}^{S}$, the . 
$k$ th stable homotopy group of spheres. This means that, for any $(k, l), l \leq k-2$, we have a group homomorphism $\phi_{k_{l} l}^{X}: \pi_{k}(X, *) \otimes \pi_{l}^{S} \rightarrow \pi_{k+l}(X, *)$ defined by $\phi_{k, l}^{X}=\bar{\psi}_{k, l+k}^{X}$ which is natural with respect to $X$. More generally, given $\left(k, l_{1}, \cdots, l_{r}\right)$ with $l_{1} \leq k-2, l_{2} \leq k+l_{1}-2, \cdots, l_{r} \leq k+l_{1}+\cdots+l_{r-1}-2$, we can define inductively the group homomorphism

$$
\phi_{k ; l_{1}, l_{2}, \cdots, l_{r}}^{X}: \pi_{k}(X, *) \otimes \pi_{l_{1}}^{S} \otimes \cdots \otimes \pi_{l_{r}}^{S} \rightarrow \pi_{k+l_{1}+\cdots+l_{r}}(X, *)
$$

by

$$
\phi_{k ; l_{1}, \cdots, l_{r}}^{X}=\phi_{k+l_{1}+\cdots+l_{r-1}, l_{r}}^{X}\left(\phi_{k ; l_{1}, \cdots, l_{r-1}}^{X} \otimes \mathrm{id}\right)
$$

$\phi_{k, l_{1}}^{X} \ldots, l_{r}$ is clearly natural with respect to $X$. If we apply this homomorphism to $X=\mathrm{PL}_{n} / O_{n}$ and $\mathrm{PL} / O$, and one uses the homotopy equivalence $\operatorname{Diff}\left(D^{n}, \partial D^{n}\right) \simeq$ $\Omega^{n+1}\left(\mathrm{PL}_{n} / O_{n}\right)$, we obtain

Theorem 7.1. For any $n$ and any sequence of natural numbers $k, l_{1}, \cdots, l_{r}$ with the property (*), $l_{1} \leq k-2, l_{2} \leq k+l_{1}-2, \cdots, l_{r} \leq k+l_{1}+\cdots+l_{r-1}-2$ there exists a group bomomorphism

$$
\begin{aligned}
\psi_{k ; l_{1}, \cdots, l_{r}}: \pi_{k-n-1} & \left(\operatorname{Diff}\left(D^{n}, \partial D^{n}\right)\right) \otimes \pi_{l_{1}}^{s} \otimes \cdots \otimes \pi_{l_{r}}^{s} \\
& \rightarrow \pi_{k-n-1+l_{1}+\cdots+l_{r}}\left(\operatorname{Diff}\left(D^{n}, \partial D^{n}\right)\right),
\end{aligned}
$$

so that the following diagram is commutative:

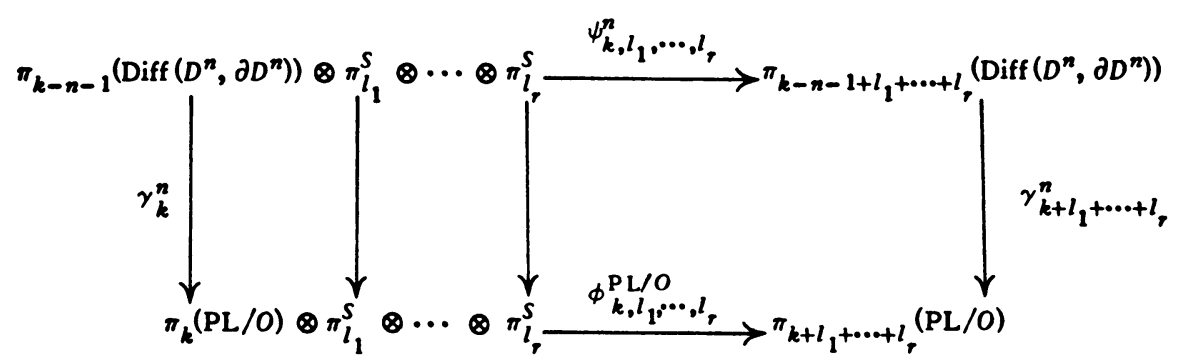

where $\gamma_{p}^{n}: \pi_{p-n-1}\left(\operatorname{Diff}\left(D^{n}, \partial D^{n}\right)\right) \rightarrow \pi_{p}(\mathrm{PL} / O)$ is the bomomorpbism. induced for bomotopy groups by $\operatorname{Diff}\left(D^{n}, \partial D^{n}\right) \simeq \Omega^{n+1}\left(\mathrm{PL}_{n} / O_{n}\right) \rightarrow \Omega^{n+1}(\mathrm{PL} / O)$.

Proof. We take $\psi_{k_{r} l_{1}, \cdots, l_{r}}^{n}=\phi_{k, l_{1}, \cdots, l_{r}}^{P L_{n} / o_{n}}$ via the isomorphism

$$
\pi_{s-n-1}\left(\mathrm{Diff}\left(D^{n}, \partial D^{n}\right)\right) \cong \pi_{s}\left(\mathrm{PL}_{n} / O_{n}\right) \text {. }
$$

Remark. For $r=1$ and $J_{l}: \pi_{l}(S O) \rightarrow \pi_{l}^{S}$ the $J$-homomorphism 
$\psi_{k, l}^{n}\left(\mathrm{id} \otimes J_{l}\right): \pi_{k-n-1}\left(\operatorname{Diff}\left(D^{n}, \partial D^{n}\right)\right) \otimes \pi_{l}(S O) \rightarrow \pi_{k-n-1+l}\left(\operatorname{Diff}\left(D^{n}, \partial D^{n}\right)\right)$ $(l \leq k-2)$ seems to be the so-called Munkres-Milnor-Novicov pairing see [2]. It seems also that $\phi_{k, l_{1}}^{\mathrm{PL} / O}$ is the so-called Bredon pairing (see [27]). As we are not interested, we do not prove the equivalence between this construction and the mentioned pairings which are originally defined in very geometric terms. In what follows we will use The orem 7.1 to get estimates about $\pi_{*}\left(\operatorname{Diff}\left(D^{n}, \partial D^{n}\right)\right)$. We should mention that John Grier Miller [28] has used the Bredon pairing (in the original Bredon definition) to detect nontrivial elements in $\pi_{*}\left(\operatorname{Diff}\left(D^{n}, \partial D^{n}\right)\right)$. His geometric point of view is different from the present, and the range of $(k, n)$ in $\pi_{k}\left(\right.$ Diff $\left.\left(D^{n}, \partial D^{n}\right)\right)$ for which he can detect nontrivial elements is essentially less than ours.

In the computations below, we will start with $k=n+2$ because for $n \geq 5$ by Theorem 4.6(Ap), $\gamma_{n+2}^{n}$ is an epimorphism so it suffices to check $\phi_{n+2, l_{1}}^{\mathrm{PL} /{ }^{2}} \cdots+l_{r}$ is nontrivial in order to conclude that $\pi_{1+l_{1}+\cdots+l_{j}}\left(\operatorname{Diff}\left(D^{n}, \partial D^{n}\right)\right)$ is nontrivial (in fact, in order to conclude $\pi_{1+l}+\cdots+l_{j}-s\left(\operatorname{Diff}\left(D^{n+s}, \partial D^{n+s}\right)\right)$ is nontrivial). For $k=n+2$ the condition (*) becomes

(**) $\quad l_{1} \leq n, \quad l_{2} \leq n+l_{1}, \quad l_{3} \leq n+l_{1}+l_{2}, \cdots, l_{r} \leq n+l_{1}+\cdots+l_{r-1}$.

Let us consider the following commutative diagram:

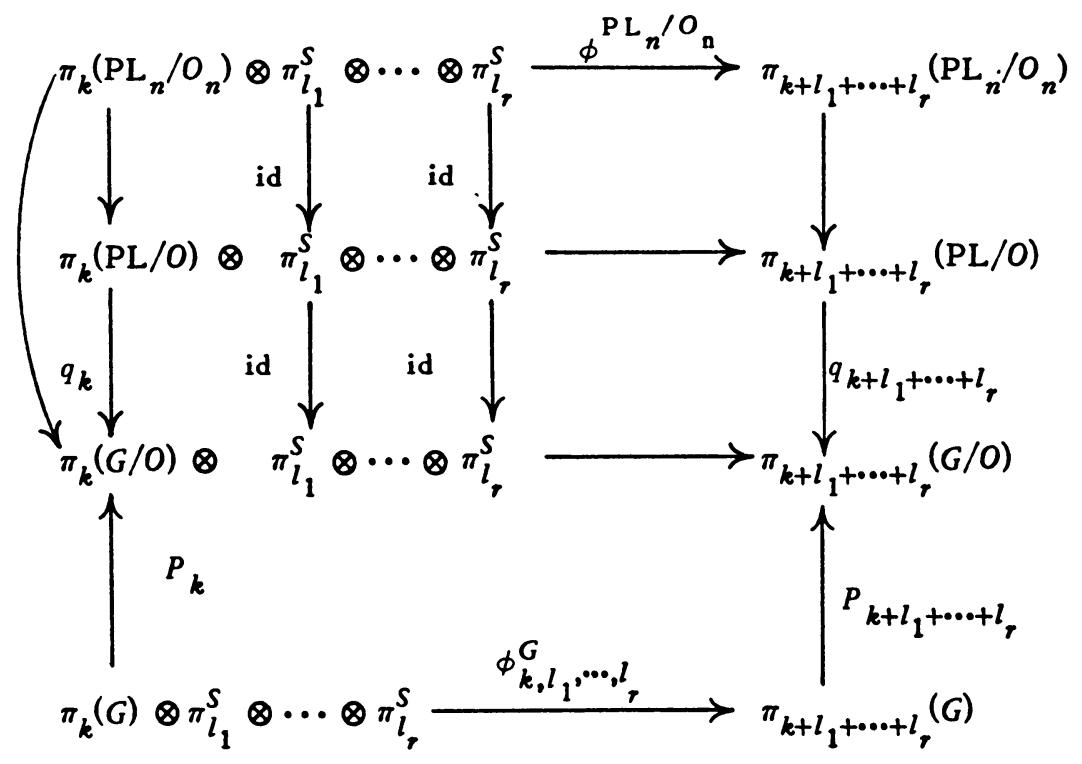

where $G$ denotes the topological semigroup $G=\operatorname{inj} \lim G_{n}$, where $G_{n}$ is the space of all continuous homotopy equivalences of $S^{n-1}$. It is well known that $\pi_{i}(G)=\pi_{i}^{S}$ and easy to check that $\phi_{k, l_{1}, \cdots, l_{r}}^{G}$ viewed as the group homomorphism 
$\phi_{k, l_{1}, \cdots, l_{r}}^{G}: \pi_{k}^{S} \otimes \cdots \otimes \pi_{l_{r}}^{S} \rightarrow \pi_{k+l_{1}+\cdots+l_{r}}^{S}$ is the standard composition (multiplication ) in $\pi_{*}^{S}$.

We also notice that $P_{k}$ is surjective for all $k \neq 0(\bmod 4)$ and $q_{k}$ is surjective $(5)(\bmod 2)$ for all $k \neq 0(\bmod 4)$ and surjective for all $k \neq 2^{l}-2$ [29].

Proposition 7.2. If $n \geq 5, n \neq 2(\bmod 4), l_{1}, \cdots, l_{r}$ is a sequence of natural numbers so that $l_{1} \leq n, l_{2} \leq n+l_{1}, \cdots, l_{r} \leq n+l_{1}+\cdots+l_{r-1}$ and $\left(P_{n+2+l_{1}+\cdots+l_{r}} \cdot \phi_{n+2, l_{1}}^{G}, \cdots, l_{r}\right) \equiv 0(\bmod 2)$ or $n \neq 2^{i}-4$ and $P_{n+2}+l_{1}+\cdots+l_{r}$. $\phi_{n+2, l_{1}, \cdots, l_{r}}^{G} \neq 0$, then

$$
\pi_{1+l_{1}+\cdots+l_{r}-s}\left(\operatorname{Diff}\left(D^{n+s}, \partial D^{n+s}\right)\right) \neq 0 .
$$

Moreover, $\gamma_{n+2+l_{1}+\cdots+l_{r}}^{n+s} \neq 0$ for any $s<1+l_{1}+\cdots+l_{r}$

It is clear that it suffices to prove Proposition 7.2 only for $s=0$ because $\gamma_{n+2+l_{1}}^{n}+\cdots+l_{r} \neq 0$ implies

$$
\pi_{1+l_{1}+\cdots+l_{r}}\left(\mathrm{PL}_{n} / O_{n}\right) \rightarrow \pi_{1+l_{1}+\cdots+l_{r}}(\mathrm{PL} / 0)
$$

is different from zero and therefore

$$
\pi_{1+l}+\cdots+l_{r}\left(\mathrm{PL}_{n+s} / O_{n+s}\right) \rightarrow \pi_{1+l_{1}+\cdots+l_{r}}(\mathrm{PL} / 0)
$$

is different from zero, which is equivalent to saying $\gamma_{n+2+l_{1}+\cdots+l_{r}}^{n+s} \neq 0$. The proof for $s=0$ is immediate from the hypotheses.

To make Propoșition 7.2 effective, we recall the following theorem of Toda [24].

Theorem 7.3.(a) For any prime number $p$ there exist $\alpha_{1} \in\left(\pi_{2 p-3}^{s} ; p\right)$ and $\beta_{t} \in\left(\pi_{2(t p+t-1)(p-1)-2}^{s} ; p\right), 1 \leq t \leq p$, so that

$$
\left(\pi_{2(r p+s+1)(p-1)-2(r-s)-1}^{s} ; p\right)=Z_{p}=\left\{a_{1} \cdot \beta_{1}^{r-s-1} \cdot \beta_{s+1}\right\}
$$

for $0 \leq s<r \leq p-1$ and $r-s \neq p-1$, where $(G, p)$ denotes the p-primary component of the abelian group $G$ and $\{x\}$ means that the group $\{x\}$ is generated by $x$.

(b) If $p \neq 2$ then $P_{2(r p+s+1)(p-1)-2(r-s)-1}\left(a_{1} \cdot \beta_{1}^{r-s-1} \cdot \beta_{s+1}\right) \neq 0$.

We take now $p \neq 2, s=0, n=2 p(p-1)-4, l_{1}=2 p-3, l_{2}=l_{3}=\cdots=l_{r}=$ $2 p(p-1)-2, \beta_{1} \in \pi_{2 p(p-1)-2}^{s}=\pi_{n+2}^{s}, a_{1} \in \pi_{2 p-3}^{s}$, and then we know

(5) If $h: G_{1}-G_{2}$ is a group homomorphism, $G_{1}, G_{2}$ abelian groups, we say " $h$ is surjective mod 2 " if the cokernel of $h$ belongs to the class of 2-primary groups and we say $h$ is "zero mod 2" if its image belongs to the class of 2-primary groups. 


$$
P_{n+2+l_{1}+\cdots+l_{r}} \cdot \phi_{n+2, l_{1}, \cdots, l_{v}}^{G}\left(\beta_{1} \cdot \alpha_{1} \cdot \beta_{1} \cdots \beta_{1}\right) \neq 0(\bmod 2) .
$$

Theorem 7.4. If $p$ is an odd prime, there exists a subgroup

$$
\mathrm{Z}_{p} \subseteq \pi_{2 p+2(r-1)\left(p^{2}-p-1\right)-2-s}\left(D_{\text {iff }} D^{2\left(p^{2}-p-2\right)+s}, \partial D^{2\left(p^{2}-p-2\right)+s}\right)
$$

sucb that

$$
\begin{aligned}
\gamma::: / Z_{p}: & Z_{p} \rightarrow \pi_{2 p+2 r\left(p^{2}-p-1\right)-3}(\mathrm{PL} / 0) \\
& \rightarrow \Theta_{2 p+2 r\left(p^{2}-p-1\right)-3} / b P_{2 p+2 r\left(p^{2}-p-1\right)-2}
\end{aligned}
$$

is nontrivial, therefore injective, for any $1 \leq r \leq p-2$ and any $s<2 p+$ $2(r-1)\left(p^{2}-p-1\right)-2$.

Proof. It suffices to prove Theorem 7.4 for $s=0$ because this means

$$
\begin{aligned}
\left.\mathrm{Z}_{p} \subseteq \pi_{2 p+2 \pi\left(p^{2}-p-1\right)-3^{(P L}}{ }_{2\left(p^{2}-p-2\right)} / 0_{2\left(p^{2}-p-2\right)}\right) \\
\quad \rightarrow \pi_{2 p+2 r\left(p^{2}-p-1\right)-3}(\mathrm{PL} / 0) \rightarrow \Theta_{2 p+2 r\left(p^{2}-p-1\right)-3} / 6 P 2 p+2 \pi\left(p^{2}-p-1\right)-2
\end{aligned}
$$

is injective, and since the first arrow factors through

$$
\pi_{2 p+2 \pi\left(p^{2}-p-1\right)-3}\left(\mathrm{PL} 2\left(p^{2}-p-2\right)+s^{2\left(p^{2}-p-2\right)+s}\right)
$$

we get Theorem 7.4 in its full generality. The proof for $s=0$ follows immediately from Proposition 7.2 and Theorem 7.3 as soon as we notice that the element $\phi^{\mathrm{PL}_{n}{ }{ }^{\prime O}{ }_{n}}\left(\tilde{\beta}_{1}, a_{1}, \beta_{1}, \cdots, \beta_{1}\right)$ has to be of order $p$, because $\beta_{1}$ is. $\tilde{\beta}_{1}$ is an element in $\pi_{n+2}\left(\mathrm{PL}_{n} / O_{n}\right)$ which by $q_{n+2} \gamma_{n+2}^{n}$ goes onto $P_{k}\left(\beta_{1}\right)$.

Theorem 7.3 is of particular interest for $r=p-2$; in which case we know that

$$
\pi_{2\left(p^{3}-4 p^{2}+3 p+2\right)-s} \operatorname{Diff}\left(D^{2\left(p^{2}-p-2\right)+s}, \partial D^{\cdots}\right)
$$

contains elements of order $p$ which survives in $\theta_{2 p\left(p^{2}-3 p+2\right)-1}$. These elements give the first known examples of nontrivial elements in $\pi_{k}\left(D\right.$ iff $\left.D^{n}, \partial D^{n}\right)$ which survives in $\Theta_{n+k+1}$ for $k>n$.

Remarks. (a) Of course other computations in stable homotopy theory which are compatible with the conditions required by Proposition 7.2 can be successfully used.

(b) The method described cannot be used for detecting elements in $\pi_{k}\left(D\right.$ iff $\left.\left(D^{n}, \partial D^{n}\right)\right)$ which go by $\gamma_{k}^{n}$ in $b P_{n+k+2}$ (because of the homotopy structure of $G / \mathrm{PL})$. 
We can use the result stated by Theorem 7.4 to get nontrivial elements in $\pi_{i}\left(\operatorname{Diff}\left(M^{n}\right)\right)$ for quite general manifolds. For instance, combining Theorem 7.4 with the diagram of $[3$, p. 128] with 3.5 .13 we obtain the following.

Theorem 7.5. Let $M^{n}$ be a compact differentiable manifold wbicb is a bomotopy torus. Then for any $p \neq 2$ and $r<p-1$ so that $n=2\left(p^{2}-p-2\right)+s$ witb $s<2 p+2(r-1)\left(p^{2}-p-1\right)-2$,

$$
\pi_{2 p+2(r-1)\left(p^{2}-p-1\right)-2-s}\left(\operatorname{Diff}\left(M^{n}, X\right)\right)
$$

contains elements of order $p$.

As a general conclusion about Theorem 7.4, we would mention that given $n$, $\pi_{*}\left(D\right.$ iff $\left.\left(D^{n}, \partial D^{n}\right)\right)$, the homotopy of the connected component of identity, contains elements of order $p \notin 2$ if $n \geq 2\left(p^{2}-p-1\right)$.

\section{SUPPLEMENTARY REFERENCES. II}

26a. A. Haefliger and C. T. C. Wall, Piecewise linear bundles in the stable range, Topology 4 (1965), 209-214. MR 32 \#1716.

27. G. Bredon, $A \pi_{*}$-module structure for $\boldsymbol{\theta}_{*}$ and applications to transformation groups, Ann. of Math. (2) 86 (1967), 434-448. MR 36 \#4570.

28. J. G. Miller, Homotopy groups of diffeomorphism groups (preprint).

29. W. Browder, The Kervaire invariant of framed manifolds and its generalizations, Ann. of Math. (2) 90 (1969), 157-186. MR 40 \#4963.

30. M. G. Barratt and M. Mahowald, The metastable homotopy of $O(n)$, Bull. Amer. Math. Soc. 70 (1964), 758-760. MR 31 \#6229.

31. I. James, On the iterated suspension, Quart. J. Math. Oxford Ser. (2) 5 (1954), 1-10. MR 15, 891.

32. Mark Mahowald, Some Whitehead products in $S^{n}$, Topology 4 (1965), 17-26. MR 31 \#2724.

33. G. F. Paechter, The groups $\pi_{r}\left(V_{n, m}\right)$ I, II, III, IV, Quart. J. Math. Oxford Ser. (2) 7 (1956), 249-268; ibid. 9 (1958), 8-27; 10 (1959), 17-37; 241-260. MR 24 \#A1725; 25 \#4540; \#4541; \#4542.

34. C. Rourke and B. Sanderson, Block bundles. I, Ann. of Math. (2) 87 (1968), 1-28. MR 37 \#2234a.

35. I. A. Volodin, Algebraic K-theory, Uspehi Mat. Nauk 27 (1972), no. 4 (166), 207208. (Russian) (to appear).

$\longrightarrow$ Generalized Whitehead groups and pseudo-isotopy, Uspehi Mat. Nauk

36. R. J. Milgram, On the Haefliger knot groups, Bull. Amer. Math. Soc. 78 (1972), 861-865.

37. A. Chenciner, Pseudooisotopies différentiables et pseudo-isotopies linéaires par morceaux, C. R. Acad. Sci. Paris Sér. A-B 270 (1970), A1312-A1315. MR 42 \#2489. 
38. M. Hirsch, On tubular neighborhoods of piecewise linear and topological manifolds, Conference on the Topology of Manifolds (Michigan State Univ., E. Lansing, Mich., 1967), Prindle, Weber and Schmidt, Boston, Mass., 1968, pp. 63-80. MR 38 \#5226.

39. N. Kuiper and R. Lashof, Microbundles and bundles. I, II, Invent. Math. 1 (1966), 1-17, 243-259. MR 35 \#7339; \#7340.

40. G. Brumfiel, On the homotopy groups of $B P L$ and PL/O, Ann. of Math. (2) 88 (1968), 291-311. MR 38 \#2775.

MATHEMATICAL INSTITUTE OF THE ROMANIAN ACADEMY, BUCHAREST, ROMANIA

DEPARTMENT OF MATHEMATICS, UNIVERSITY OF CHICAGO, CHICAGO, ILLINOIS 60637 